\title{
New algorithms for reducing cross-dispersed echelle spectra ${ }^{\star}$
}

\author{
N. E. Piskunov ${ }^{1}$ and J. A. Valenti ${ }^{2}$ \\ 1 Department of Astronomy and Space Physics Uppsala University, Box 515, 75120 Uppsala, Sweden \\ 2 Space Telescope Science Institute 3700 San Martin Dr. Baltimore, MD 21218, USA \\ e-mail: valenti@stsci.edu
}

Received 1 November 2001 / Accepted 31 January 2002

\begin{abstract}
We describe advanced image processing algorithms, implemented in a data analysis package for conventional and cross-dispersed echelle spectra. Comparisons with results from other packages illustrate the outstanding quality of the new REDUCE package, particularly in terms of resulting noise level and treatment of CCD defects and cosmic ray spikes. REDUCE can be adapted relatively easily to handle a variety of instrument types, including spectrographs with prism or grating cross-dispersers, possibly fed by a fiber or image slicer, etc. In addition to reduced spectra, an accurate spatial profile is recovered, providing valuable information about the spectrograph PSF and simplifying scattered light corrections.
\end{abstract}

Key words. instrumentation: spectrographs - methods: data analysis - techniques: spectroscopic

\section{Introduction}

Cross-dispersed echelle spectrographs are now in widespread use, providing broad wavelength coverage at high dispersion in a rectangular format well suited to pixel array detectors. Commissioning of the UltravioletVisual Echelle Spectrograph (UVES) at the Very Large Telescope (VLT) facility inspired this exploration of procedures for reducing raw echelle data into calibrated one-dimensional spectra.

Techniques for reducing cross-dispersed echelle spectra have been described by many authors (e.g. Moreno et al. 1982; Rossi et al. 1985; Ponz et al. 1986; Goodrich \& Veilleux 1988; Marsh 1989; Mukai 1990; Hall et al. 1994; Valenti 1994; Piskunov 1995; Hinkle et al. 2000). Despite this rich cumulative heritage, it is important to formulate mathematically the concept of the best solution for each step involved and use it to critically analyze and improve the existing algorithms. We adopt several requirements for an ideal echelle reduction package.

Cross-dispersed echelle orders are curved and hence cannot be aligned with detector rows or columns. Raw data should not be interpolated to straighten echelle orders (as in Moreno et al. 1982) because this distorts the extracted spectrum (Marsh 1989; Mukai 1990) and leads

\footnotetext{
Send offprint requests to: N. Piskunov,

e-mail: piskunov@astro.uu.se

* Based on data obtained with the VLT UVES and SAAO Giraffe spectrometers.
}

to cyclical variations in $\mathrm{S} / \mathrm{N}$ ratio and effective instrumental profile. Order curvature and location should be determined empirically from the actual image or at least from contemporaneous data.

Each spectral order is a two-dimensional intensity surface built from a sequence of one-dimensional monochromatic slit images. These images can be averaged to produce a relatively low noise one-dimensional model that can be used to "optimally" extract an order. Optimal extraction maximizes $\mathrm{S} / \mathrm{N}$ ratio in the extracted spectrum and more importantly provides a statistical basis for identifying and handling bad pixels.

Data reduction should be driven by a flexible scripting language, but otherwise require minimal interaction. Automated procedures are less subjective, yield reproducible results, and allow batch processing of large datasets. Conditions that are not handled automatically must be detected and reported, so that problems can be traced easily and fully processed spectra are always of the highest quality.

Using the Interactive Data Language (IDL), we have developed the software package REDUCE to process crossdispersed echelle or even single order data. Our REDUCE package is derived from the Batch Echelle Reduction Package (BERP), written mainly by Valenti and described in Hinkle et al. (2000). BERP evolved from procedures described in Valenti (1994), adding support for several spectrographs and implementing a form of optimal extraction inspired by Piskunov (1995). 
The optimal extraction algorithm in REDUCE only works when the relative illumination profile perpendicular to echelle dispersion (hereafter "spatial profile") is aligned with detector rows or columns and varies slowly with wavelength. Aside from these limitations, REDUCE handles a wide variety of situations, including single order spectra, closely packed echelle orders, fiber or slicer input, undersampled images, noisy data, etc.

In this paper we provide a brief overview of REDUCE and then describe in detail new algorithms developed mainly by Piskunov for mapping order locations and determining spatial profiles used in optimal extraction. When appropriate, we describe the behavior of REDUCE by reference to existing literature. We illustrate performance using red-arm UVES (VLT, D'Odorico 2000) and GIRAFFE (SAAO, MUSICOS-type fiber-fed instrument) spectra of the rapidly oscillating peculiar A-type (roAp) stars HD 217522 (UVES) and HD 166473 (GIRAFFE), as well as a blue-arm UVES spectrum of the metal poor subgiant CS 22892-052. We compare output from REDUCE, BERP, IRAF, and the ESO MIDAS/UVES reduction.

\section{Overview of the reduction process}

All raw calibration and science data must be in FITS format. A modular routine reads images, trims extraneous pixels, and forces a canonical orientation with wavelength in each order increasing left to right and order number increasing top to bottom. A mean bias frame is subtracted from all non-bias images.

Certain instrument and exposure specific information is required for processing, including orientation of the echelle format, usable portion of the detector, gain, read noise, background count rate, and exposure time. REDUCE either extracts these quantities (if available) from the FITS header or uses default values for the specified instrument. Values may also be mandated in the reduction script for a particular set of data. This information is then written back to the FITS header using E_ keywords, such as E_TIME, which contains the exposure time. Creation of standardized header keywords simplifies communication between procedures and allows most routines to be instrument independent, making it relatively easy for proficient spectroscopists with IDL programming experience to add support for new instruments.

Calibration data should include bias, flat field, and wavelength exposures. Incandescent lamp exposures obtained with a cross-dispersed echelle spectrograph are not at all "flat", but we use this standard terminology nonetheless. Flats with different exposure times may be used to optimize $\mathrm{S} / \mathrm{N}$ ratio in the blue and red. Optional calibration data may include dark frames and an order definition image (often a flat field obtained with a short aperture).

All calibration data for an observing sequence are processed before the science data. Bias frames are co-added in two groups (preferably before and after science observations). The pair of co-added bias frames are compared
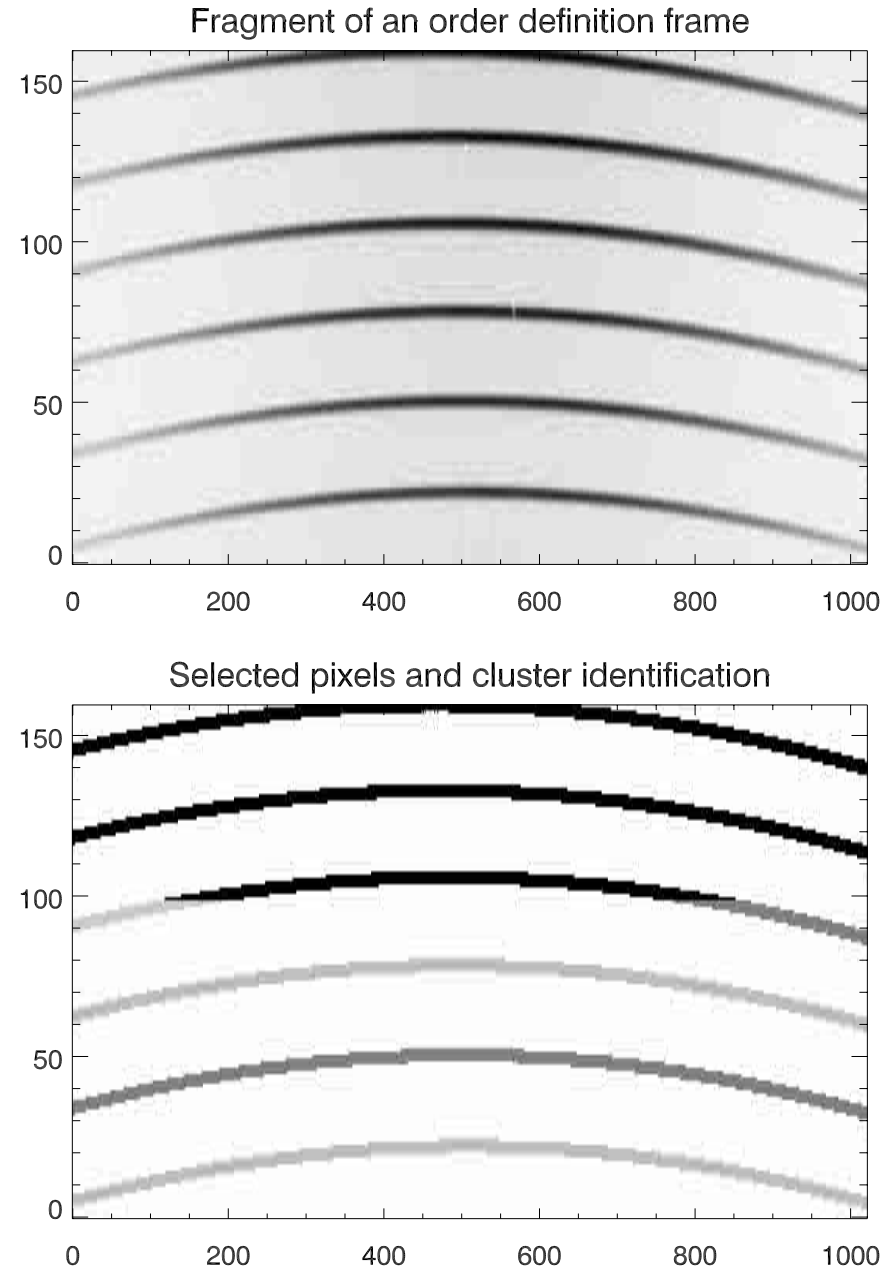

Fig. 1. Illustration of the cluster "coloring" procedure. The top panel shows a negative of the original image on a logarithmic scale. This spectrum of HD 166473 was taken with the GIRAFFE fiber-fed spectrometer (SAAO). The shapes of orders are defined by the prism cross-disperser. The bottom panel shows every pixel selected by criterion (1) and a snapshot taken during the cluster coloring process. The three bottom orders have been grouped into three distinct clusters. As identification proceeds upwards, the next order begins as two separate clusters that will eventually merge into one cluster. The top two orders have not yet been painted.

to detect bias shifts, monitor read noise, and identify outliers. The two summed bias images are then combined into a mean bias frame, ignoring bad pixels.

Construction of a mean flat field image requires more care because lamp brightness can vary, longer exposures are more susceptible to cosmic rays, and echelle orders may drift between exposures (especially if they bracket science observations). Pixel by pixel median filtering of normalized images handles lamp variations and cosmic rays, but fails when orders drift. Rather than treating pixels independently, we analyze a group of several rows (or columns depending on spectrum orientation). Intensity may change rapidly along a row, but we assume slow spatial changes in the relative intensity of different flat field exposures. In the limit of negligible drift, relative 

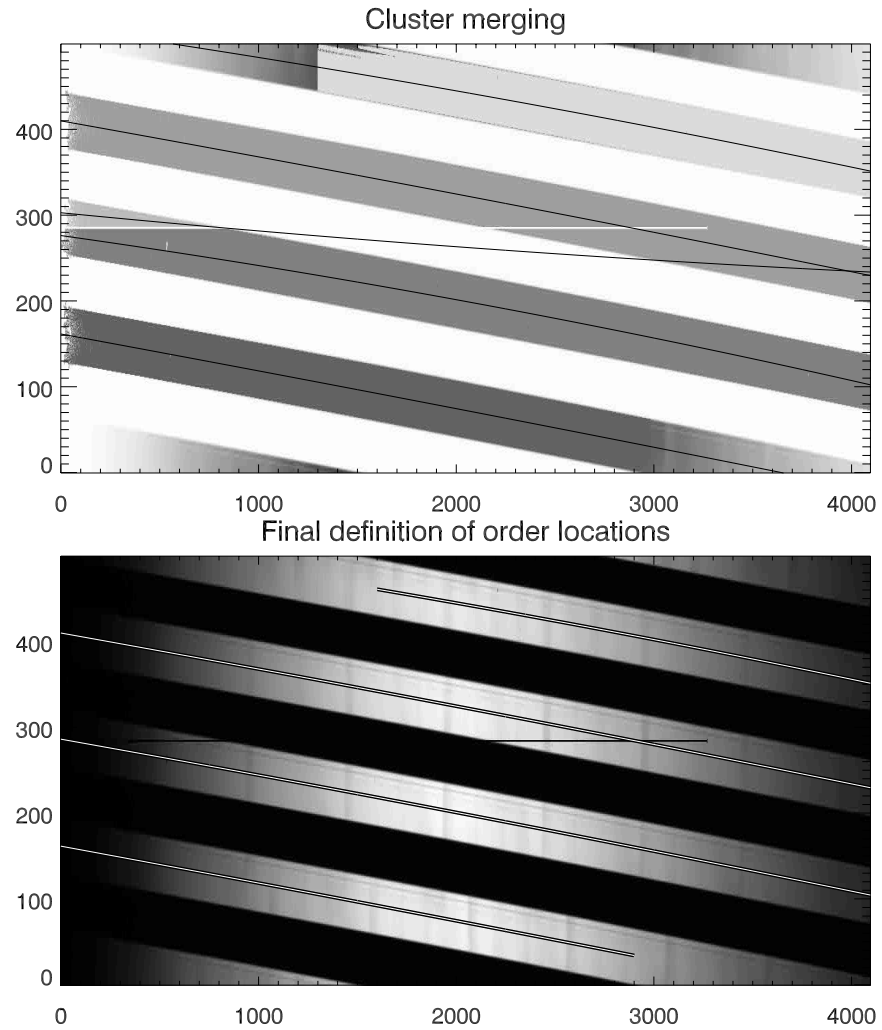

Fig. 2. The cluster identification (coloring) and merging procedure. The top panel shows (in negative) 7 clusters detected in the image. The two small corner clusters are ignored, as they have pixels in the top or bottom row for all $x$. The other two partial orders are restricted to columns where they do not touch end rows of the detector. The black lines trace order location fits. A bad row (white line around row 300) cuts the third order into two clusters. The fit to the smaller cluster has a higher coincidence with the fourth order and will confuse the automatic merging procedure. The interactive mode remedies the situation, as shown in the bottom panel.

intensity is constant over the entire image, but the procedure allows for moderate spatial variations.

The information from neighboring pixels in a row is combined to obtain a precise estimate of the relative intensity distribution expected for any individual pixel. The expected intensity distribution can be used in conjunction with a noise model to identify all statistically significant outliers, due to cosmic rays or other abnormal processes. The distribution also provides weights for combining uncorrupted pixels. This procedure is essentially a variant of optimal extraction, used mainly to identify and remove bad pixels. See Hinkle et al. (2000) for more details. After co-addition, the mean flat field image is normalized, leaving only pixel-to-pixel quantum efficiency variations and fringes. In Sect. 6 we describe the procedure we use to normalize the flat field image.

In Sect. 3 we describe a new clustering algorithm that robustly locates spectral orders in two-dimensional spectroscopic data. A well-exposed order definition image is used to map default order locations, which are then used for extracting spectra with negligible continuum (e.g. wavelength calibration exposures) or even for all spectra if the spectrograph is stable. Order locations may also be traced for individual stellar spectra.

Science data are initially processed by subtracting the mean bias image and then dividing by the normalized flat. A low noise spatial profile is determined along each order, and an empirical noise model is constructed for each pixel. This information is used to optimally extract each order by fitting the spatial profile to each column in an order. Formal uncertainties are computed for each extracted pixel. The measured response function along orders ("order shape") in the flat field image are used to approximately flatten each extracted order.

\section{Mapping order locations}

In general echelle order curvature and location on the detector can either be assumed a priori or determined empirically from the image being reduced. Assumed order maps can be calculated from an optical model or measured empirically from a reference image. Alignment of the assumed map with actual order locations may require an offset. Although some echelle spectrographs may be stable enough to rely on an assumed order map, we believe a general echelle reduction package should determine order curvature and location empirically, whenever possible.

Empirical order maps may be determined interactively or automatically. Automated procedures are preferable because they are reproducible and allow batch processing of many spectra, though the robustness of human interaction must then be captured in an automated algorithm. Automated procedures must also evaluate whether order mapping has been successful, halting or reverting to a default order map if a specific image cannot be mapped, for example when there is no signal.

The new order location algorithm presented here consists of four steps: selection of pixels that might be in spectral orders, clustering analysis of these selected pixels, merging and rejection of clusters, and fitting of merged clusters. The procedure is sufficiently robust to use on every science image, but UVES is stable enough that order locations need only be determined once, for example using the mean flat. A special order definition image may be needed when an image slicer is used as the entrance slit.

Recall that after forcing a canonical orientation, spectral orders are aligned approximately along image rows. Selection of pixels that might be in spectral orders is done by first smoothing each column and then selecting pixels above the median of the difference between the original and the smoothed column:

$a_{x, y}$ is selected if $a_{x, y}>\bar{a}_{x, y}+\operatorname{MEDIAN}\left(a_{x, y}-\bar{a}_{x, y}\right)$

The smoothing filter can be tuned depending on order separation and contrast. Figure 1 shows an example of pixels selected by the criterion in Eq. (1).

The $x$ and $y$ indices of selected pixels are stored and used in the clustering analysis, which associates connected 


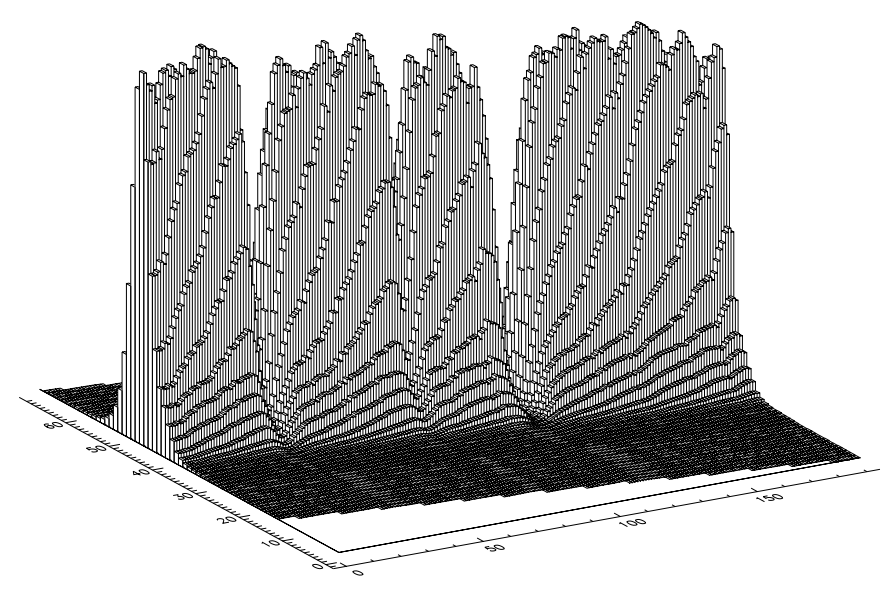

Fig. 3. Pixel sampling of a spectral order is usually optimized to use detector area efficiently, while retaining adequate sampling. This minimal sampling results in visible "interference" between periodic pixel spacing and quasi-periodic spacing of columns where orders cross from one detector row to the next. This is seen as "waves" in the maximum amplitude along an order. The large depressions in this fragment of the UVES CS 22892-052 spectrum are absorption lines.

groups of pixels. One can think of this procedure as coloring selected pixels so that all neighboring pixels (with $x$ and $y$ that differ by at most 1) have the same color. This is a non-trivial problem because some initially detached clusters may ultimately need to be merged, as illustrated in Fig. 1.

Coloring begins by creating a vector to store the color of each selected pixel in $x$ and $y$. Neighbors in the same row are easily detected by sorting $x$ and $y$ so that $y$ increases monotonically, while $x$ scans the row corresponding to each value of $y$. In order to make the procedure as efficient in the vertical direction, we also sort $x$ and $y$ so that $x$ increases monotonically, while $y$ scans columns. We use a lookup array that relates both sets of sorted coordinates to assign a common color to neighboring pixels, thereby forming a cluster. Spurious clusters may persist in areas of very low signal. After the coloring step, clusters with fewer pixels than some tunable threshold are deselected, causing them to be ignored in the final fitting step.

Orders are often truncated by the top or bottom edge of the detector, potentially hampering attempts to fit order location. To avoid this problem, columns with cluster pixels in the first or last row of the detector are ignored, when fitting the remaining pixels in a cluster (see Fig. 2). This prepares for accurate extraction of partial orders.

Even after clustering analysis, a single order may be partitioned into multiple clusters due to detector defects or deep absorption features. Polynomial fits are useful for deciding whether to merge or discard clusters. For each cluster, we fit a polynomial of tunable order to pixel $y$ values, as a function of $x$, and then extend the fit to cover all columns. Extended fits for pairs of consecutive clusters are compared to identify which pair has fits that are most coincident for $x$ values present in the later cluster. As a

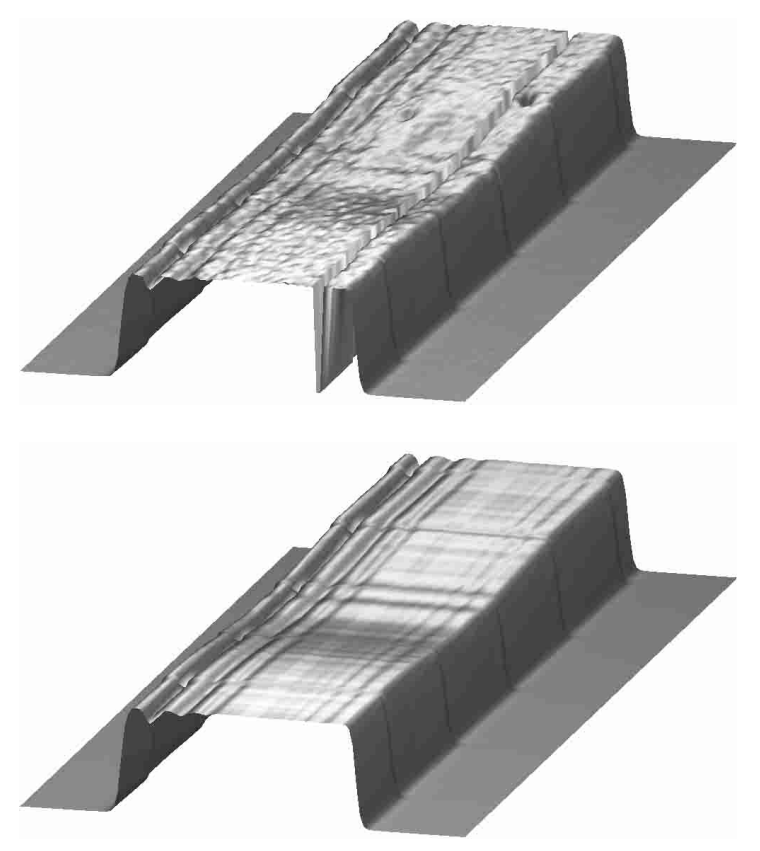

Fig. 4. A segment of a flat field order (top) and the corresponding model image (bottom) reconstructed from the decomposed spectrum and oversampled $(M=10)$ spatial profile. The flat field image was obtained with the ESO UVES spectrometer using an image slicer. Note the bad row and the low sensitivity pixel (a trap) near the right edge of the observed order. In the model reconstruction the bad row has been completely removed with the help of a mask, and the trap is automatically removed by the decomposition algorithm.

metric of coincidence, we use the number of columns in which both fits disagree by less than a few pixels.

In automatic mode, the most coincident pair of clusters is merged if the two fits coincide over at least $95 \%$ of the $x$ values present in the later cluster. Otherwise, the smaller of the two clusters is ignored. After merging or discarding a cluster, coincidence is re-assessed based on new polynomial fits. Merging halts when no clusters coincide. This procedure must converge in less than $N_{\text {clusters }}-N_{\text {orders }}$ iterations but in practice the detector properties and layout of the focal plane may allow more rapid convergence.

If the automated merging algorithm fails, manual intervention is required. The only situation we found requiring such intervention was the case of a bad row crossing multiple spectral orders (Fig. 2). In this case, fits to partial clusters created by the bad row may result erroneously in more significant coincidence with an adjacent order.

For each order, our clustering analysis yields a polynomial description of order location, an uncertainty estimate for the fitted polynomial, and the beginning and ending columns to use during spectrum extraction. In addition, we attempt to identify the echelle order number of the first and last order in the image, based on the observed change in order spacing. For a grating cross-disperser, the grating equation for the echelle (assuming that each 

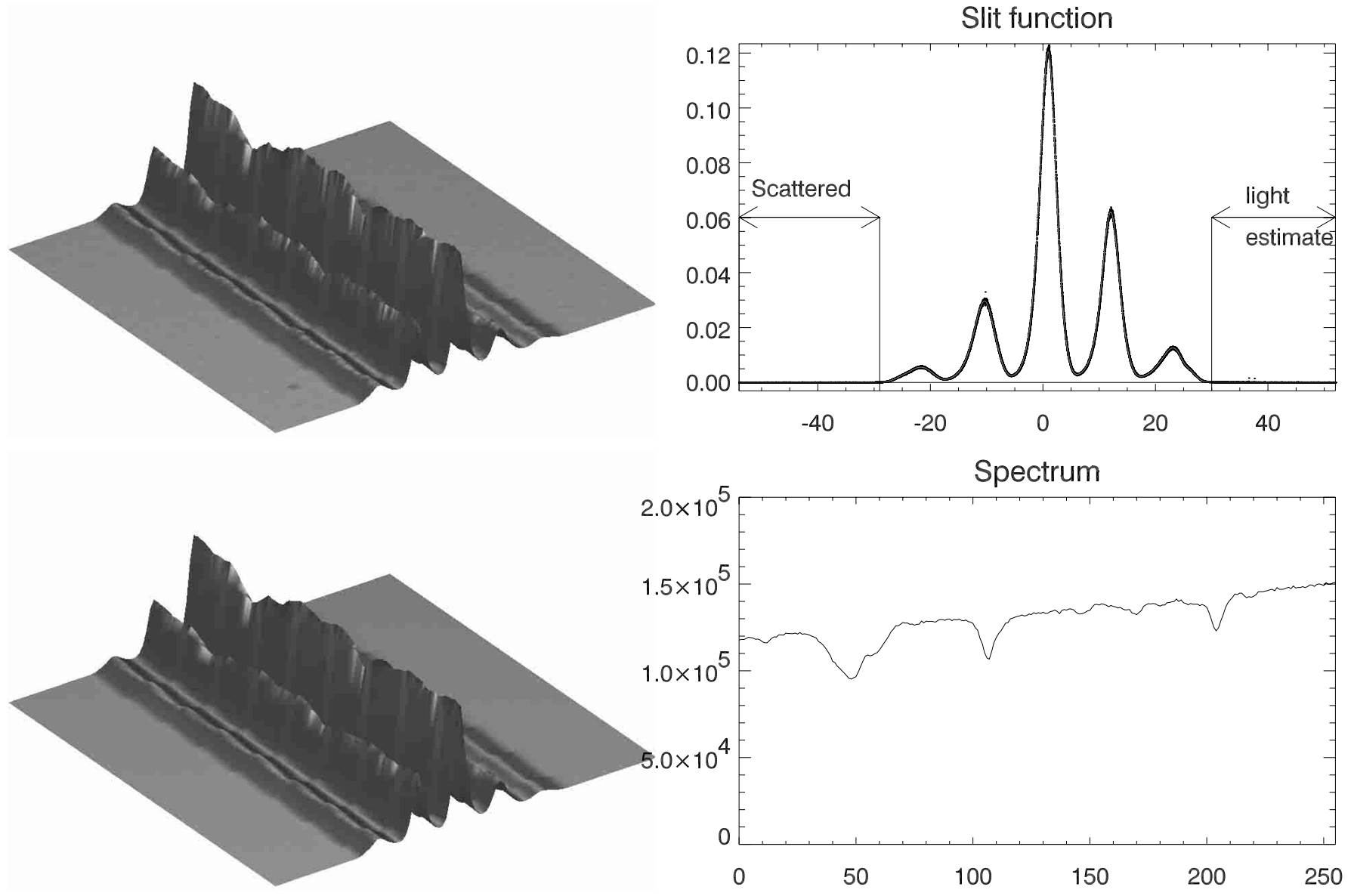

Fig. 5. A segment of a stellar spectrum (HD 217522) obtained with an image slicer (upper left) and the corresponding decomposition into an oversampled $(M=10)$ spatial profile (upper right) and the spectrum (lower right). The reconstructed model image (Eq. (4)) is shown in the lower left. The upper-right panel also shows data for every column, scaled and offset according to the model. The only significant outlier corresponds to a cosmic ray feature in the observation which left no trace in the extracted spectrum. The regions used to estimate scattered light are marked in the upper-right panel.

detector column corresponds to a constant reflection angle) gives:

$\Delta y \cdot n \cdot(n+1)=$ const.

where $\Delta y$ is the separation between echelle orders $n$ and $n+1$ along a fiducial column on the detector. An analogous relationship can be defined for prism cross-dispersers. In principle, three consecutive orders are sufficient to determine $n$, even without knowledge of the constant. In practice, echelle spectrographs operate at very high $n$, making Eq. (2) rather similar for adjacent order pairs. Nonetheless, echelle order identification is usually reliable for the large number of orders typically present in many echelle spectrographs.

\section{Spatial and spectral decomposition of an order}

Perhaps the most important recent improvement in echelle reduction procedures is the introduction of spectral order decomposition. Recall that we define the spatial profile to be the relative illumination profile of an order perpendicular to echelle dispersion. For an ideal optical system that is perfectly aligned, the spatial profile is a monochromatic image of the illuminated entrance slit, aligned along detector columns (after re-orientation, if necessary). In this case a spectral order $S$ can be represented as the product of a spectrum $f$ and a spatial profile $g$ :

$S(x, y)=f(x) \times g\left(y-y_{0}(x)\right)$

where the function $y_{0}(x)$ describes the order location. In practice, the ideal image is also convolved with the PSF and sampled with detector pixels of significant size (Fig. 3). Although detector pixels set a natural spatial scale, $g$ must be sampled on a finer grid to reproduce the sampling "waves" visible in Fig. 3.

The discrete version of Eq. (3) includes integration of the spatial profile over each detector pixel:

$S_{x, y} \approx f_{x} \cdot \sum_{j} \omega_{x, y}^{j} g_{j}$

where $\omega_{x, y}^{j}$ are the weights, which differ from zero only for indices $j$ that map into pixel $x, y$. The structure of the $\omega$ for each column deserves a closer look. Define an integer oversampling factor, $M$, to be the detector pixel size divided by the grid size adopted for $g$. Then $\omega_{x, y}^{j}$ for 

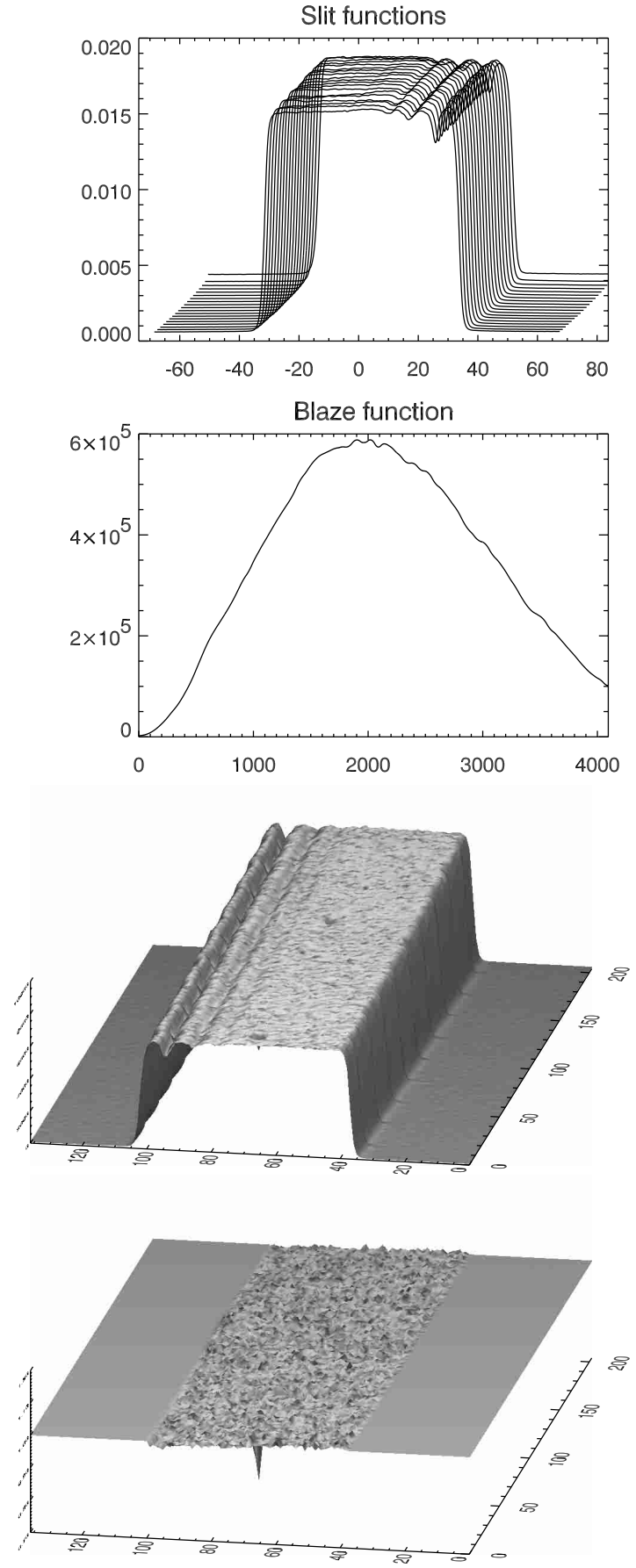

Fig. 6. Flat field normalization using spatial profiles. Panels from the top: spatial profile for individual swaths, the derived order shape function, a segment of the mean flat field image containing part of a single order, and the same segment after normalization using spatial profiles.

a given $x, y$ is:

$$
\omega_{x, y}^{j}= \begin{cases}0 & j<j_{0} \\ \mathbf{F R A C T}\left(y_{0}(x) M\right) & j=j_{0} \\ 1 / M & j=j_{0}+1, \ldots, M-1 \\ 1-\operatorname{FRACT}\left(y_{0}(x) M\right) & j=j_{0}+M \\ 0 & j>j_{0}+M\end{cases}
$$
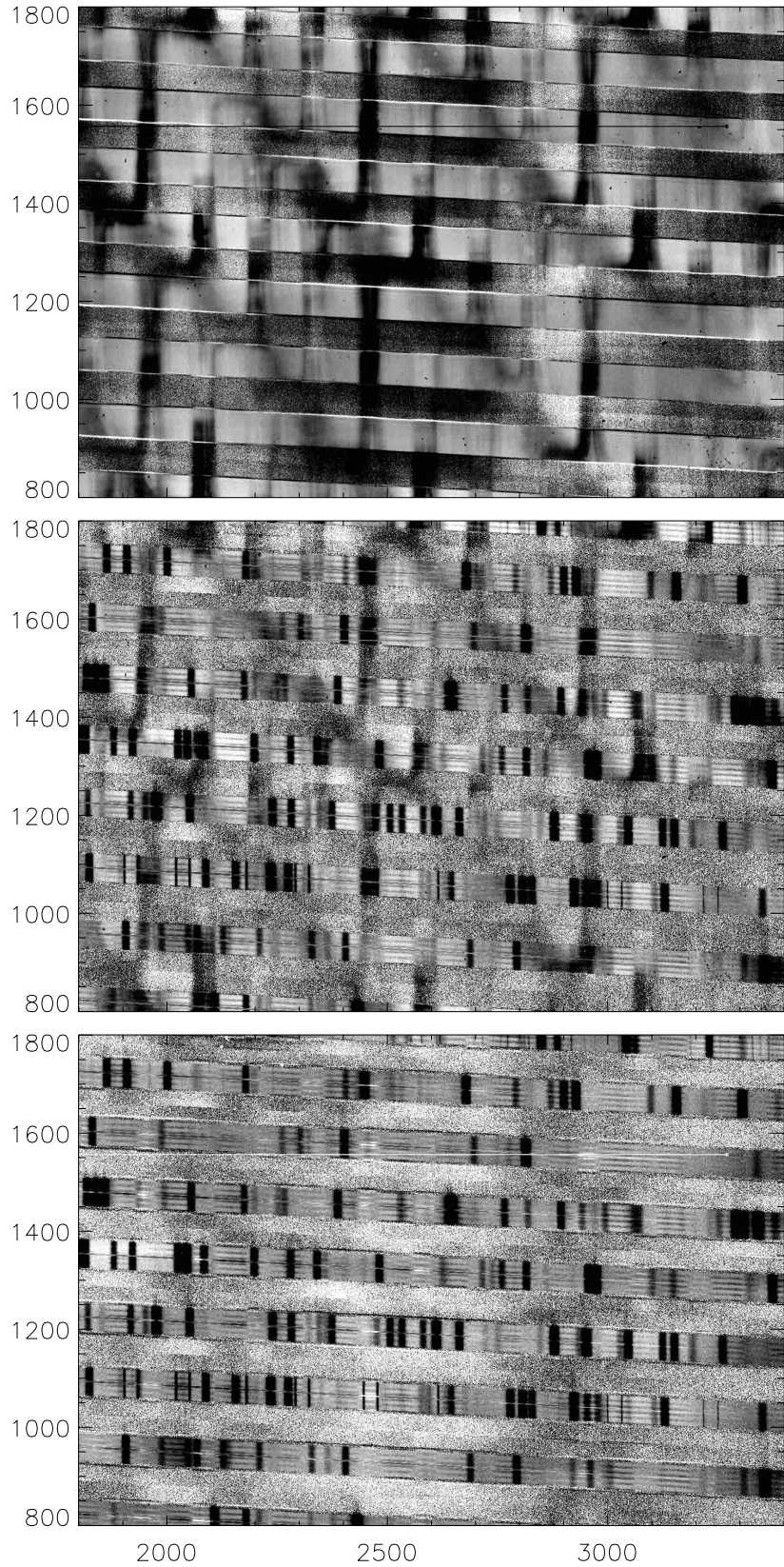

Fig. 7. Flat fielding of stellar spectra. The top panel shows a normalized flat field image. Vertical and diagonal bands of reduced sensitivity are likely caused by CCD internal structure. The middle panel shows a normalized stellar spectrum (HD 217522), for illustration purposes only, since this is not part of the normal reduction procedure. The bottom panel shows a flat fielded stellar spectrum image.

where $j_{0}$ is the index of the first subpixel that falls (perhaps only partially) in detector pixel $x, y$, and FRACT is the fraction of that first subpixel that is contained in the detector pixel. This structure is exactly the same for all $y$ at a given $x$ ! The relationship between the $j_{0}$ and $j_{0}+M$ elements of $\omega$ reflects the periodic nature of $\omega$, due to the use of an integer oversampling factor.

During spectroscopic reduction, our goal is to decom(5) pose each observed order $S_{x, y}$ into the spectrum $f_{x}$ and a 
spatial profile $g_{j}$. We can accomplish this decomposition by solving the inverse problem:

$\mathcal{F} \equiv \sum_{x, y}\left[f_{x} \sum_{j} \omega_{x, y}^{j} g_{j}-S_{x, y}\right]^{2}=$ minimum.

The main advantage of this approach is that the number of data points exceeds the number of unknowns for all practical problems. To avoid an obvious scaling ambiguity for $f$ and $g$, we require $g$ to be area normalized.

Selection of the oversampling factor $M$ needs special attention. One extreme case occurs when orders are perfectly aligned with detector rows, in which case no oversampling $(M=1)$ is needed. As the derivative of $y_{0}(x)$ deviates from zero, $M$ should increase. In order to decouple the value of $M$ from varying order inclination (thereby making the problem tractable), we introduce an additional constraint: (6):

$\mathcal{F}+\Lambda \sum_{j}\left(g_{j+1}-g_{j}\right)^{2}=$ minimum.

The second term smooths the spatial profile, restricting point-to-point variations, even if the order geometry does not constrain every point in $g$. In order to solve the restricted problem (7) we set the derivatives of $g$ and $f$ equal to zero. This produces two systems of linear equations:

$\sum_{j}\left(\mathbf{A}_{j k}+\Lambda \cdot \mathbf{B}_{j k}\right) g_{j}=\mathbf{R}_{k}$

$f_{x}=\mathbf{C}_{x} / \mathbf{D}_{x}$

with matrices given by:

$$
\begin{aligned}
& \mathbf{A}_{j k}=\sum_{x, y} f_{x}^{2} \omega_{x, y}^{j} \omega_{x, y}^{k} \\
& \mathbf{R}_{k}=\sum_{x, y} S_{x, y} f_{x} \omega_{x, y}^{k} \\
& \mathbf{C}_{x}=\sum_{y} S_{x, y} \sum_{j} g_{j} \omega_{x, y}^{j} \\
& \mathbf{D}_{x}=\sum_{y}\left[\sum_{j} g_{j} \omega_{x, y}^{j}\right]^{2}
\end{aligned}
$$

where $\mathbf{B}_{j k}$ is a tri-diagonal matrix with -1 on both subdiagonals and 2 on the main diagonal, except for the upper-left and the bottom-right corners, which contain 1 . In the matrix equations above, we use the generic nomenclature $N=N_{y} \cdot M$.

The very simple structure of $\omega_{x, y}^{j}$ makes it easy to construct the matrix $\sum_{y} \omega_{x, y}^{j} \omega_{x, y}^{k}$ for every $x$. This blockdiagonal matrix is the key to efficient computations. Each square block has $M+1$ elements on a side and is proportional to the product of two scalars, such as $\omega_{x, 0}^{j} \cdot \omega_{x, 0}^{k}$, for example. Therefore $\omega$ does not have to be computed for every value of $x$. Only the first $M+1$ elements are needed! The regular shape of $\omega_{x, y}^{j} \cdot \omega_{x, y}^{k}$ greatly simplifies the evaluation of matrix $\mathbf{A}_{i j}$ in Eq. (8), making it possible to use fast and efficient numerical methods to solve for the spatial profile.

Iteration is used to solve Eqs. (8)-(13). Beginning with an initial guess for the spectrum (e.g. $f_{x}=\sum_{y} S_{x, y}$ ), we solve Eq. (8) to obtain the spatial profile, normalize the total area, and then solve Eq. (9) to obtain an improved estimate of the spectrum. Iteration ceases when the maximum fractional change in the spectrum becomes small.

The decomposition procedure produces the best possible spectrum and slit function, if the order location $y_{\mathrm{o}}(x)$ is accurately determined and large scale structure in the image is dominated by the spectrum. Local defects like cosmic rays, bad pixels, and noise have minimal impact on the results! This can be illustrated by comparing an observed order with a model order constructed from the derived $f$ and $g$, convolved with detector pixels according to Eq. (4) (Figs. 4 and 5). The only type of artifact that adversely affects decomposition are rows of bad pixels with length and orientation similar to spectral orders. Fortunately, such defects can usually be identified a priori and ignored during decomposition, leaving no trace of the bad row (Fig. 4).

Generalization of the decomposition procedure to handle tilted or curved slit images is straightforward, if the image geometry is known. The only difference is that the mapping between $f$ and detector pixel is a function of both $x$ and $y$, making the pattern of $\omega_{x, y}^{j}$ slightly more complicated. Nevertheless, the structure of $\mathbf{A}_{j k}$ remains block-diagonal. If the PSF has a 2D structure, for example when an image slicer is used, the order can still be decomposed using a 2D representation of the PSF in place of the 1D spatial profile. For UVES, deviations from a 1D representation of $g$ cause errors smaller than the typical readout noise.

REDUCE uses the decomposition routine to (a) normalize flat field images and derive order shape functions, (b) locate the inter-order gaps and estimate scattered light, and (c) extract spectral orders.

\section{Estimating scattered light}

Flat field normalization and spectral extraction both require estimates of the scattered light background beneath each spectral order. The spectrum itself precludes direct measurement of the background beneath an order, but interpolation of the background between orders often provides an adequate estimate.

First we locate the position and extent of background regions between orders. This step can be non-trivial if the spectrum is obtained with an image slicer. We decompose a central group of columns in each order, empirically measuring noise from the standard deviation of the observed order minus the reconstructed model (Fig. 5). We then fit a linear background to the bottom envelope of the recovered spatial profile, using a threshold that allows 


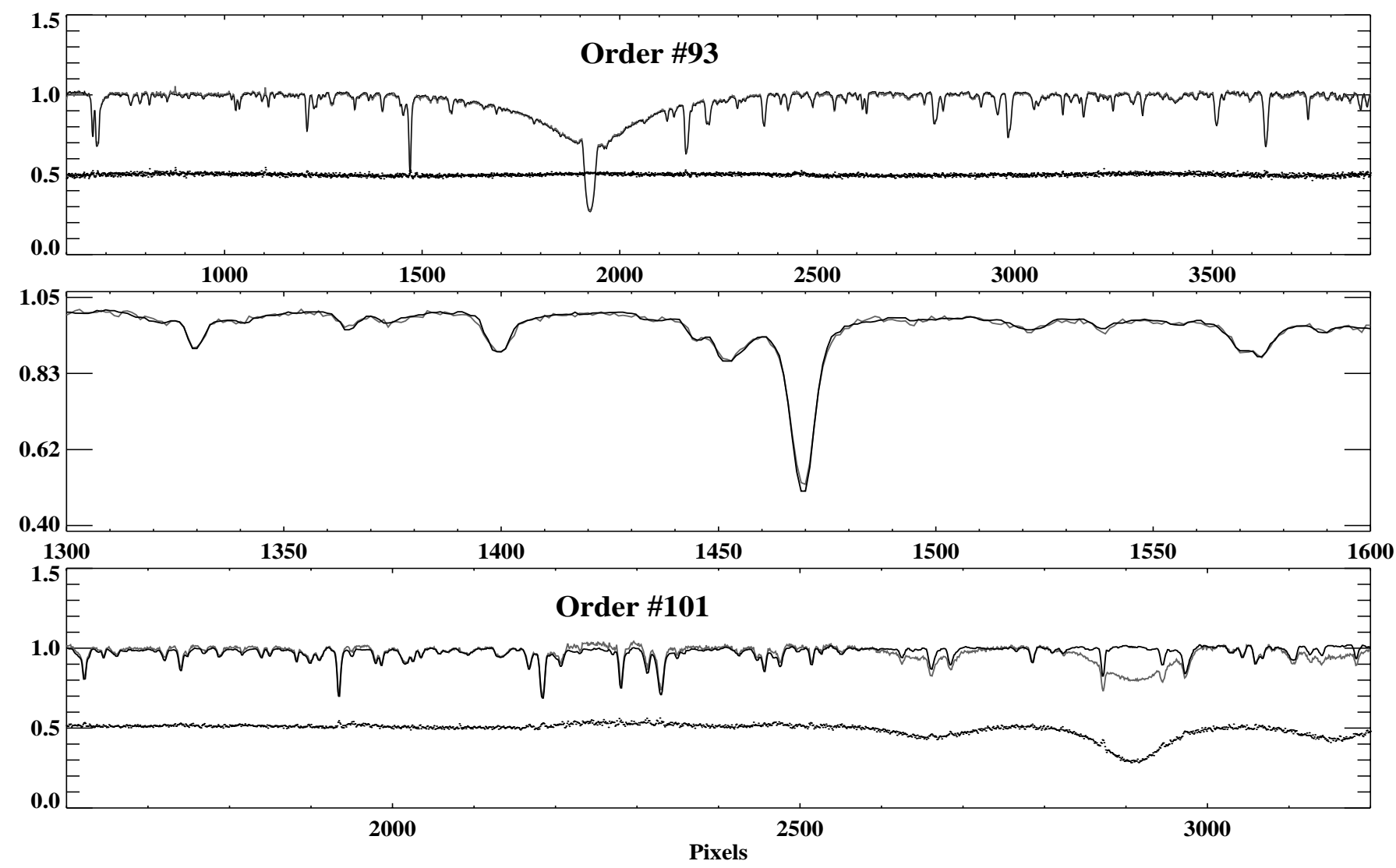

Fig. 8. Comparison of spectra of HD 217522 optimally extracted with REDUCE (narrow black line) and reduced with IRAF (wider grey line). Top panel: continuum normalized order 83 containing $\mathrm{H} \alpha$. Dots show the difference between the two reductions, shifted upwards by 0.5 . Middle panel: segment of the same order in more detail. Bottom panel: extracted spectra and difference for order 101.

background points to fall below the fit within the estimated noise level.

We define a background region at the beginning and another at the end of each spatial profile. Each background region contains several consecutive detector pixels below the linear fit just described. Robust estimation of a smooth background typically requires at least 8 pixels in a background region. This assessment is based on comparisons of background determinations in adjacent columns. A more elaborate procedure may be required to handle ghosts or scattered light from bright emission lines. Currently, sky emission is included in the background estimate and hence gets subtracted automatically from the target spectrum. If an image slicer is used, we do not attempt to use pixels between the slices to estimate background because the amount of overlap is very hard to estimate.

Background intervals defined with respect to the spatial profile are mapped into background stripes in the observed image by applying the selected offsets to the nominal order location $y_{0}$. For each column, separate medians are calculated for background pixels above and below each order. The resulting pair of background vectors for each order are filtered in the dispersion direction to remove vertical defects. Finally, linear interpolation in $y$ is used to estimate the scattered light beneath each pixel of the spectral order.

\section{Normalizing flats and determining order shapes}

Flat fielding helps remove pixel-to-pixel sensitivity variations, but care is required to minimize noise contributions from the mean flat described in Sect. 2. In particular, the signal level outside the central part of the order falls rapidly, so division by the mean flat field image would strongly magnify the contributions by low-signal (high-noise) regions in the science image. This effect is less important when the spatial profile is relatively flat, as in Figs. 4 and 5, but even in this case grooves in the left part of the order (Fig. 4) correspond to gaps between slices where the signal is weak in the science frame (Fig. 5). The problem is much more severe when the flat field images have a spatial profile similar to the science exposure, for example with fiber-fed spectrometers.

To properly weight regions at all signal levels, the mean flat field image should be normalized prior to use in calibrating science images. Models of each order, constructed from decomposed spatial profiles and extracted spectra, provide an excellent template for flat field normalization. The normalization region may be set to unity outside the useful part of the science exposure. Normalization of the flat field image is performed one order at a time. Each order is split into several parts (swaths) containing approximately identical number of columns. After decomposition 

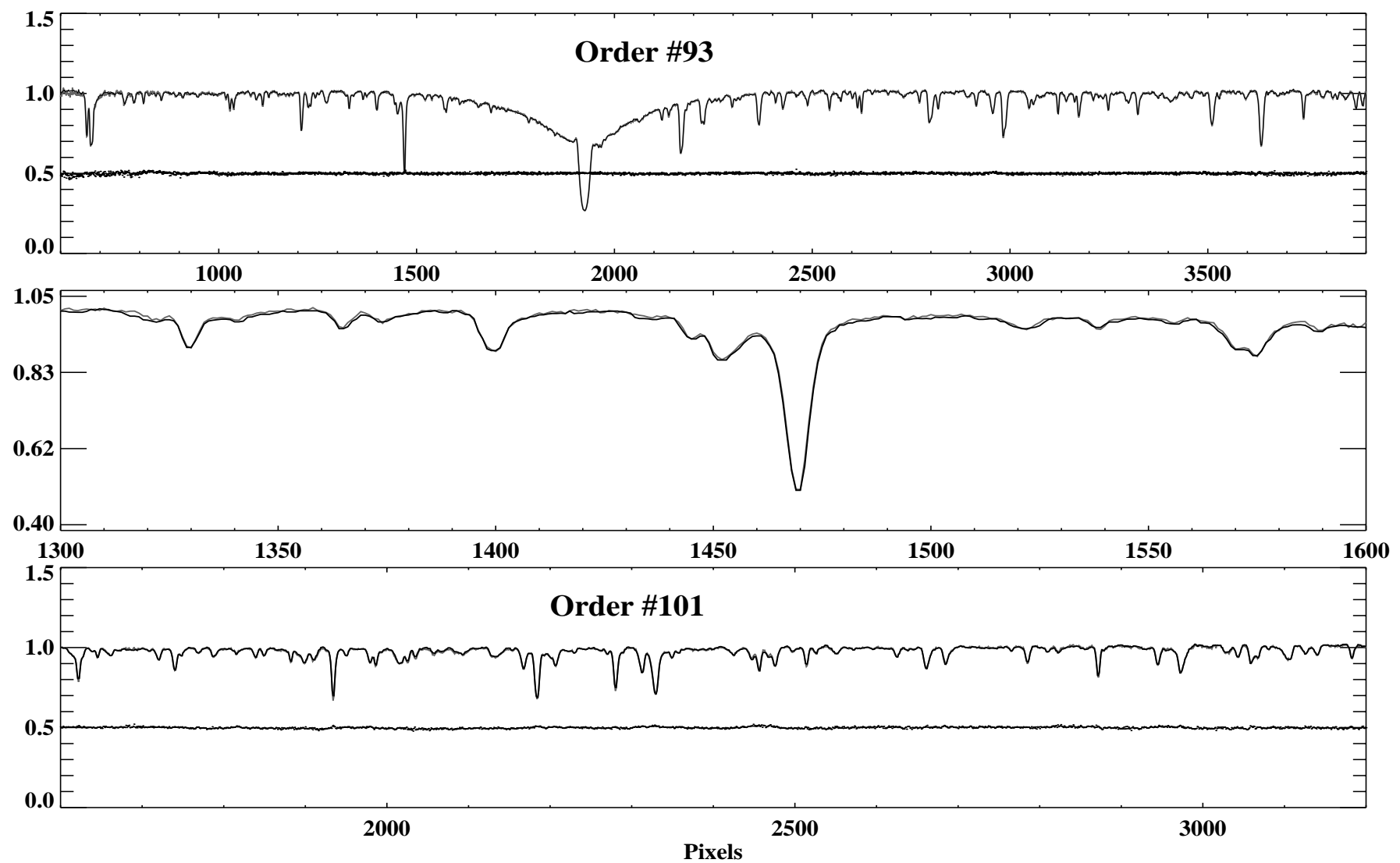

Fig. 9. Comparison of optimal extraction performed with REDUCE (narrow black line) and BERP (wider grey line) of the roAp star HD 217522 taken with an image slicer. Top panel: normalized order 83 containing H $\alpha$. Dots show the difference between the two reductions, shifted upwards by 0.5. Middle panel: segment of the same order in more detail. Bottom panel: extracted spectra and difference for order 101.

the low noise spatial profiles and spectra are stored for each swath. Spectra for all swaths are spliced together to form the shape function for each order. For each column, spatial profiles for the two nearest swaths are linearly interpolated (or extrapolated) and scaled according to the order shape function. This scaled spatial profile is then used to normalize the corresponding column segment in the mean flat. Areas outside the useful science regions are set to unity. Figure 6 shows spatial profiles for a sequence of swaths, an order shape function, and a segment of a flat field image before and after normalization.

Variations in the spatial profile must be extrapolated for pixels in the outside half of the first and the last swath. The number of columns in a swath can be specified explicitly or selected automatically by REDUCE. In the latter case, the number of columns is chosen to enforce multiple row crossing within a swath to sample the spatial profile well. At the same time, the number of swaths should be large enough to track any changes in the PSF along an echelle spectral order.

\section{Optimal extraction}

Horne (1986) introduced the concept of optimal extraction for low order spectra nearly aligned with detector rows or columns. Horne divides all pixels corresponding to the same wavelength by an estimate of the extracted spectrum at that wavelength. Processing all pixels in this manner yields a two-dimensional image of the spatial illumination profile for each wavelength. Horne fits low order polynomials perpendicular to the spatial axis, creating a relatively low-noise model of migration and changes in the spatial profile. Low order polynomials cannot describe migration of echelle orders, which cross many detector rows or pixels. Since the procedure requires knowledge of the extracted spectrum, iteration may be necessary.

Marsh (1989) modified the optimal extraction algorithm of Horne (1986) to handle echelle orders that cross many detector rows or columns, defining a set of low-order polynomials parallel to the curved echelle order being extracted. By fitting along echelle orders, polynomials need only describe changes in the spatial profile, not migration of the spectrum across detector rows or columns. However, interpolation of the raw data is required to apportion flux in neighboring pixels to polynomials with subpixel offsets. Ideally, optimal extraction should not require interpolation.

Piskunov (1995) developed an orthogonal form of optimal extraction, fitting the spatial profile for each wavelength with an empirically determined mean spatial profile. Piskunov constructed the mean spatial profile by 

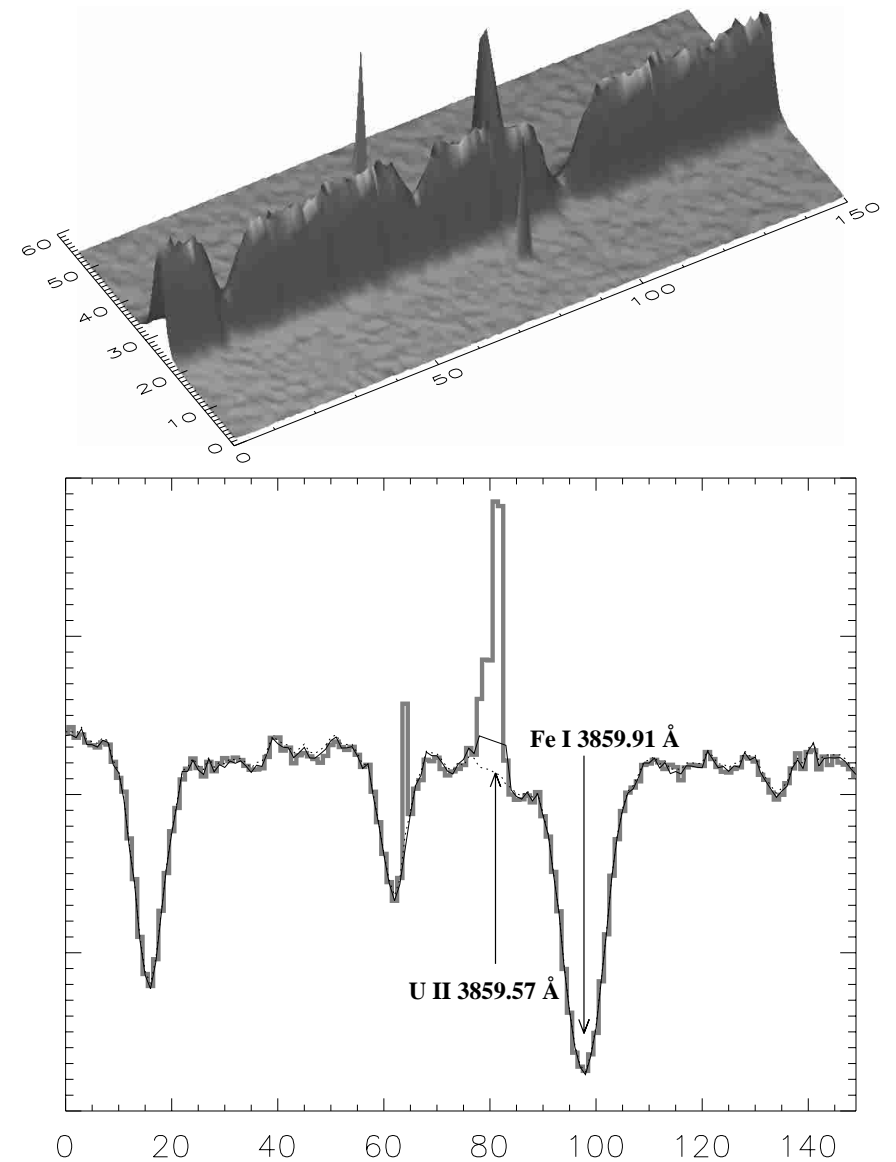

Fig. 10. Top panel: blue UVES subimage in the $3860 \AA$ region of a metal poor star CS 22892-052 taken without slicer. Bottom panel: comparison of spectra extracted with REDUCE (dashed black line), the ESO MIDAS/UVES extraction (solid black line), and a simple sum of all rows in the subimage (wide grey line).

spline interpolating spatial profiles for each wavelength onto an oversampled grid, allowing alignment and averaging despite order curvature. Hinkle et al. (2000) aligned spatial profiles for each wavelength without interpolating, keeping track of subpixel offsets. Spatial profiles were interleaved according to subpixel offsets, median filtered to remove cosmic rays, and smoothed to reduce noise. Hinkle et al. (2000) determined mean spatial profiles at several positions along the order to track changes.

Optimal extraction in REDUCE follows the same swathbased procedure introduced in the previous section. After bias subtraction, the science image is divided by the normalized flat field image. For each order, the central swath is decomposed to obtain a preliminary spatial profile used only to define background regions between orders. Background vectors above and below each order are then interpolated linearly to estimate the background in every row containing spectrum. After background subtraction, the image is decomposed again to obtain spatial profiles for every swath. Interpolation (or extrapolation) yields the spatial profile appropriate for each column in an order.
Decomposition also identifies bad pixels by assuming a constant spatial profile within a swath. Each column in an order is fit with the appropriate spatial profile, using weights based on a noise model. The resulting scale factor is the optimally extracted relative intensity of the spectrum for that order and column. To flatten the continuum, each extracted order is usually divided by the order shape function determined during normalization of the mean flat field image. For orders truncated by the top or bottom edge of the image, no extraction occurs outside the valid column intervals identified during the order location process.

The new algorithm achieves signal-to-noise ratios very close to the Poisson limit, while remaining relatively insensitive to artifacts in the data. Figure 7 illustrates one extreme case which was encountered while reducing the red-arm UVES spectra registered with an MIT CCD. The original image shows the imprint of the internal structure of the detector, which is clearly revealed in the normalized flat (Fig. 7, top panel). The amplitude of this structure reaches several percent, which is much larger than pixelto-pixel sensitivity variations. If a similar normalization procedure is applied to the science frame (middle panel), the same detector structure is revealed. Flat fielding (division of the science frame by the normalized flat) completely removes the artifacts. Alternative approaches to the optimal extraction (e.g. analytical models for the slit function) would be less successful, as the CCD structure affects science and flat field images differently. In particular, features that cross spectral orders at an angle introduce a tilt to the local cross-dispersion profiles.

\section{Performance}

The two new algorithms introduced here (clustering analysis and spatial/spectral decomposition) require significant computing power. For example, tracing 30 spectral orders on a $4096 \times 2048 \mathrm{CCD}$ takes about $2 \mathrm{~min}$ on a $500 \mathrm{MHz}$ Pentium III PC or 1 min on an HP J7000 workstation running at $544 \mathrm{MHz}$. The most time-consuming step is the decomposition routine, even with optimal use of IDL vector operations. Decomposition of a $400 \times 100$ pixel array takes about $10 / 5 \mathrm{~s}$ on the same machines. About half of this time is spent on solving the band diagonal system of equations. A call to an external routine reduces the execution time by at least $30 \%$ but makes the package platform dependent. Complete reduction of a single $2048 \times 2048$ science exposure with 20 orders, including supplementary processing of 20 flat field images, 20 bias frames, and one ThAr reference frame, takes about 1.5-2 hours on a J7000 workstation with 1 Gbyte of RAM. Processing of subsequent science exposures that share the same calibration data requires 35-40 min per frame.

\section{Comparison with other reduction packages}

We investigate performance of various algorithms, comparing spectra extracted with REDUCE, the ESO 
MIDAS/UVES context (Ballester et al. 2000), IRAF (Tody 1986), and BERP (Hinkle et al. 2000). For all packages, we use identical order locations (from the procedure described in Sect. 3) and the same normalized flat, isolating differences due mainly to choice of extraction algorithm.

To compare our optimal extraction procedure with IRAF and BERP, we consider orders 93 and 101 in a red spectrum of the roAp-star HD 217522, taken by S. Hubrig with UVES as part of program 65.I-0644 (Cowley et al. 2001). These data were taken with image slicer \#3 (5 slices). Based on the number of counts per pixel, we expect to achieve a $\mathrm{S} / \mathrm{N}$ ratio of about 250 in the extracted spectrum. Data from the red arm of UVES can be relatively challenging to reduce because of interference fringes and also the non-uniform response of the MIT CCD. We expect the various algorithms to differ mainly in residual noise, small-scale deviations due to detector defects and cosmic ray spikes, and large-scale (tens of pixels) deviations due to fringing and CCD structure. Treatment of these maladies all depend directly on the quality of slit function reconstruction. In order to assess the relative noise performance of each reduction procedure, we compute residuals with respect to spectra extracted by REDUCE, and we compare noise in the REDUCE spectrum with expectations based on photon counting statistics. To facilitate intercomparison, we map all continua onto the REDUCE output using a fourth order polynomial scaling.

At the time of this analysis we did not have a VLT/UVES pipeline reduction or a complete set of calibration data for HD 217522. Instead we examined the $3860 \AA$ region of a blue spectrum of a metal-poor star CS 22892-052 $([\mathrm{Fe} / \mathrm{H}]=-3.1)$ taken without an image slicer during UVES commissioning. We selected this star because it illustrates well the impact reduction procedures have on our ability to achieve scientific goals of current interest. Measurements of thorium and uranium abundance allow an accurate estimate of the age of CS 22892-052 (see Cayrel et al. 2001 for details). Unfortunately only the U II $3859.6 \AA$ line can be used, since the star is too hot to contain detectable amounts of $\mathrm{U}$ I.

\subsection{Comparison with MIDAS/UVES context}

Figure 10 compares output from REDUCE and the ESO MIDAS/UVES package. Reductions with the latest version of MIDAS/UVES were provided by Vanessa Hill (ESO). In order to achieve robust automatic extraction, the MIDAS/UVES context uses an analytical fit (Gaussian) to the spatial profile. Another significant difference is that MIDAS/UVES uses an extracted spectrum of the mean flat field image to correct sensitivity variations in 1D extracted spectra, whereas REDUCE applies 2D sensitivity corrections pixel-by-pixel. Bad pixels in the science image complicate the MIDAS/UVES analysis, especially in this case where the uranium line of interest sits in the wing of a strong iron line. This particular spike is a bit mysterious because it was present in 3 out of 4 science images with a different amplitude each time. The rejection of such defects in REDUCE occurs naturally, as a byproduct of the assumed regularity in the spatial profile. The final spectrum is based on a fit of good pixels only. The MIDAS and REDUCE spectra are comparable, except where the echelle order is affected by a bad pixel. Proper treatment of such artifacts may be important if the defect falls on a critical spectral feature, as in the case of CS 22892052 (Fig. 10), where the estimate of uranium abundance depends on the quality of data reduction.

\subsection{Comparison with IRAF}

Figure 8 compares REDUCE and IRAF extractions. Noise in the difference between the two spectra is twice the level expected from Poisson statistics. The middle panel of Fig. 8 reveals that the IRAF reduction is noisier, while the bottom panel shows large-scale deviations which turn out to be associated with CCD structures similar to those shown in Fig. 7.

\subsection{Comparison with BERP}

Figure 9 compares REDUCE and BERP extractions. Algorithmic design similarities almost guarantee that the packages will yield similar results. Indeed, after tuning the parameter that controls smoothing of the BERP spatial profile, we obtain virtually identical results, with residuals smaller that the Poisson noise, confirming that practically no additional noise is introduced by our reduction procedures.

After this work was completed we discovered a paper by Kinney et al. (1991) which describes an optimal extraction procedure developed for low-dispersion IUE spectra. The paper discusses some of the same issues considered here, but their procedure uses an analytical approximation (high-order polynomials in this case) to fit spatial profiles, as is done in the UVES pipeline and MIDAS/UVES package.

\section{Conclusions}

We presented the latest version of our echelle reduction package REDUCE, concentrating mainly on two novel algorithms: clustering analysis used for detecting order locations and spatial/spectral decomposition used for optimal extraction. The package incorporating these new algorithms was tested using data from spectrographs fed by fiber or image slicer and with prism or grating crossdispersers. The new procedures proved to be robust and capable of achieving maximum signal-to-noise ratio. The IDL package is easily ported to different platforms and can be configured to handle new instruments by modifying information concentrated in a limited number of instrument-specific routines.

Comparisons with results from other reduction packages demonstrates the superior ability of REDUCE to handle 
cosmic ray spikes and CCD defects and to compensate for detector interference fringes.

Acknowledgements. We acknowledge the Kurt and Alice Wallenberg Foundation which contributed the computer equipment actively used in this project. We are also thankful to E. Stempels, V. Hill and M. Mizuno-Wiedner who reduced lots of data, some of which is used in this paper. NP would like to thank S. Hubrig and P. Mittermayer for sharing their HD 217522 and HD 166473 data. We would also like to thank A.Kaufer for many useful comments which helped to improve this paper.

\section{References}

Ballester, P., Modigliani, A., Boitquin, O., et al. 2000, Messenger, 101, 31

Cayrel, R., Hill, V., Beers, T. C., et al. 2001, Nature, 409, 691

Cowley, C. R., Hubrig, S., Ryabchikova, T. A., et al. 2001, A\&A, 367, 939
D'Odorico, S., Cristiani, S., Dekker, H., et al. 2000, Proc. SPIE, 4005, 121

Goodrich, R. W., \& Veilleux, S. 1988, PASP, 100, 1572

Hall, J. C., Fulton, E. E., Huenemoerder, D. P., Welty, A. D., \& Neff, J. E. 1994, PASP, 106, 315

Hinkle, K., Wallace, L., Valenti, J. A., \& Harmer, D. 2000, Visible and Near Infrared Atlas of the Arcturus Spectrum 3727-9300 A (San Francisco: ASP), 8

Horne, K. 1986, PASP, 98, 609

Kinney, A. L., Bohlin, R. C., \& Neill, J. D. 1991, PASP, 103, 694

Marsh, T. R. 1989, PASP, 101, 1032

Moreno, V., Llorente de Andrés, F., \& Jiménez, J. 1982, A\&A, 111,260

Mukai, K. 1990, PASP, 102, 183

Piskunov, N. 1995, CCD Spectroscopy Reduction package for PCIPS Version 2.2

Ponz, D., Brinks, E., \& D’Odorico, S. 1986, SPIE, 627, 707

Rossi, C., Lombardi, R., Gaudenzi, S., \& De Bliase, G. A. 1985, A\&A, 143, 13

Tody, D. 1986, SPIE, 627, 733

Valenti, J. A. 1994, Ph.D. Thesis, Univ. California at Berkeley 